\title{
Dynamical Frequency Assignment of WSN Based on Cloud Computing Technology
}

\author{
JING Jun-li ${ }^{\mathrm{a},{ }^{* 1}}$, YANG Jie ${ }^{\mathrm{b},{ }^{* 2}}$ \\ ${ }^{a}$ College of Science, Huaihai Industrial College, Lianyungang, China \\ ${ }^{b}$ Departmant of Communication Command, Chongqing Communication College, Chongqing, China
}

\begin{abstract}
As the rapid development of Wireless Sensor Networks(WSN), the demands of frequency rose sharply, thereby how to deal with the frequency assignment of WSN is a difficult problem. A dynamical and real-time frequency assignment system is proposed, which was based on the network architecture of Internet of Things by the means of Cloud Computing technique. The system assigned the frequency dynamically and real-timely that it can improve the frequency utilization rate. This system is an efficient path to face the frequency needs of WSN.
\end{abstract}

Index Terms: Wireless Sensor Network(WSN); Dynamical Frequency Assignment; Cloud Computing; Spectrum Sensing

(C) 2012 Published by MECS Publisher. Selection and/or peer review under responsibility of the Research Association of Modern Education and Computer Science

\section{Introduction}

Wireless Sensor Networks(WSN) is a wireless autonomous monitoring network system composed by multiple tiny sensor nodes with communication and limited computing capability distributed in the monitoring region. The demands of frequency for WSN will be great. How to achieve rational planning and distribution of frequency in the limited electromagnetic space, and how to solve the problem of shortage in frequency resources for WSN, that has a key influence on the development of future WSN[1].

The limitation of traditional static assignment of frequency is that the average utilization of frequency is very low. According to the data from Federal Communications Commission (FCC), the utilization of frequency that has been allocated is only $15 \%$ [2].So many countries strengthen the dynamic frequency assignment technology research to improve the utilization of frequency. For example, the U.S. newt Generation communications program (XG) propose a frequency management that "strategy - behavior - Agreement" mutual separation and independent of the three; Trinity College Dublin, Ireland made a living model of spectrum management system; Cognitive radio(CR) use dynamic frequency assignment techniques such as "frequency pooling system", "opportunistic access" to improve the frequency utilization. However, the studying of dynamic frequency assignment for WSN is still scarce. References [3] proposed frequency switching

* Corresponding author:

| E-mail address: ${ }^{* 1}$ lygzjj1@ sina.com; ${ }^{* 2}$ yangjie5581@163.com 
methods for WSN nodes to achieve frequency dynamic using in a small range. But it is difficult to achieve Dynamic assignment of a large number of frequency for Large-scale WSN in a wide range.

The paper proposed a dynamical frequency assignment method for WSN based on cloud computing technology. The method assigns frequency dynamically by considering the frequency needs of WSN, and the characteristics of wide range or large-scale WSN, using Cloud resource managing, distributed frequency database management, frequency analysis and decision support technologies. So this paper may provide a new way to meet the frequency demand for WSN.

\section{Dynamical Frequency Assignment for WSN}

To meet the huge frequency demand for WSN, Dynamic Spectrum Management (DSM) is one of the important solutions. In the authorized frequency band for WSN, According to the "spectrum holes " that spectrum formed in the frequency domain, geographical domain and time domain three-dimensional space and the communication quality of service (QoS) of terminal nodes, DSM provide the available non-fixed frequencies for users. In order to describe the process of dynamic frequency assignment clearly, the functional model is shown in Fig. 1.

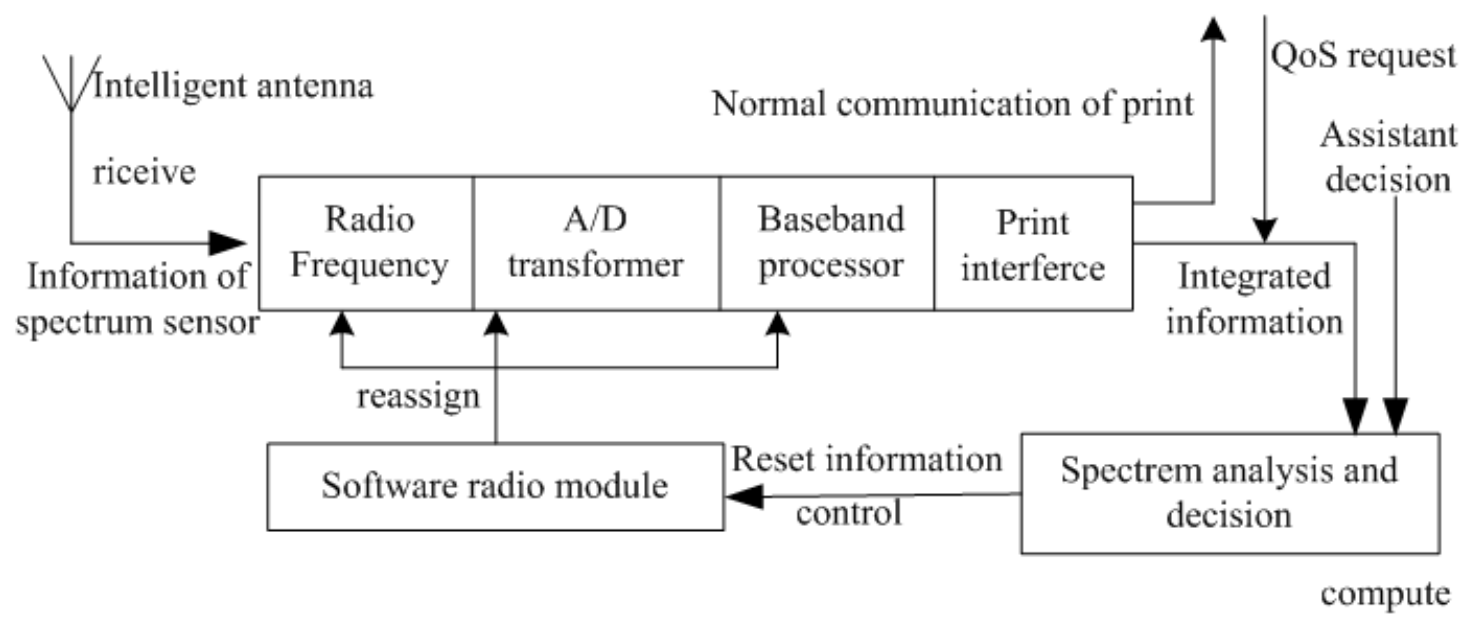

Fig. 1. DSM information function model

\section{1) Acquiring information of Spectrum sensing:}

Mainly depends on WSN nodes with spectrum sensing capabilities, Continued detecting the authorized frequency bands and surrounding electromagnetic environment in the frequency domain, geographical domain and time domain three-dimensional space to learn the using of frequency; using the way of multi-nodes association detecting to improve the reliability of spectrum detecting.

\section{2) Information Processing:}

Mainly including spectral analysis and spectrum decision based cloud computing technology; cognizing spectrum and switching frequency according detected information and QoS such as data rate, error rate, delay limits, transmission mode and bandwidth etc.

\section{3) Achieving reconfiguration:}

The terminal nodes automatically change their own frequency parameters according reconfiguration requirements. This requires nodes the reconfiguration ability in addition to the cognizing spectrum ability .All 
these nodes can adjust their own working parameters such as frequency, modulation mode, transmission power and communication technology without any hardware changing. This feature can be realized by software radio.

In XG systems, terminal equipment determine their own frequency by spectrum strategy analysis[4-5]. This is not applicable to wide range WSN because the wide range WSN have too many nodes that has no enough computing power for frequency assignment. In addition, WSN nodes often move in a wide range that these nodes must updating their own frequency when beyonding a certain range while the updating is difficult. So, establishing a dynamic frequency assignment system for WSN which can deal with all those spectrum analysis and decision may be a way. But this way face many problems such as processing large amounts of information, high real-time requirements and mass information storage etc. Existing centralized distribution system can't solve all these problems.

\section{Dynamical Frequency Assignment System of WSN Based on Cloud Computing Technology}

\subsection{System structure}

In the processing of dynamic frequency assignment for WSN, by the coordinated operation of cloud computing platform with powerful computing capabilities and those sensor nodes with sensing capabilities, dynamical frequency assignment system based on cloud computing can be established, the structure is shown in Fig. 2.

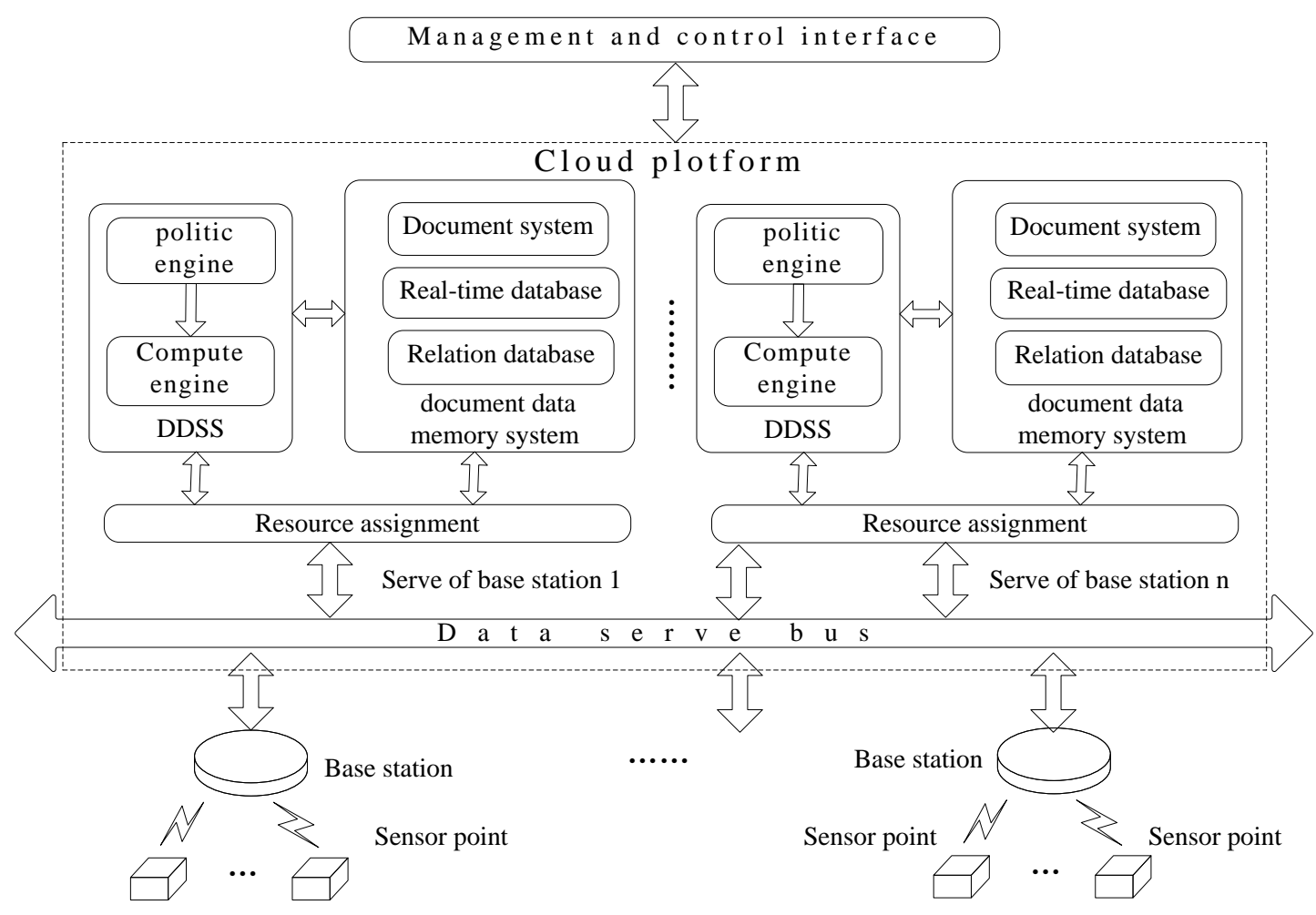

Fig. 2. Dynamical frequency assignment system structure of WSN

In the processing of dynamic frequency assignment for WSN, by the coordinated operation of cloud computing platform with powerful computing capabilities and those sensor nodes with sensing capabilities, 
dynamical frequency assignment system based on cloud computing can be established, the structure is shown in Fig. 2. The cloud computing platform is data processing center of dynamic frequency assignment for WSN which Including some core components such as data service bus, distributed data storage system, resource management strategy and the distributed decision support systems(DDSS) etc. The cloud computing platform integrates DDSS, distributed data storage system, resource management strategy by the distributed data service bus forming a efficient and reliable dynamic frequency assignment system for WSN.

Dynamical frequency assignment system based on cloud computing for WSN integrated transmission and exchange with spectrum data and frequency distribution information by applying Distributed Memory, Distributed Computing, Virtualization Technology and dynamic load balancing. The system has powerful data processing and spectrum analysis capabilities by dynamical managing virtual underlying hardware. Meanwhile the system also has a unified management and monitoring interface so we can monitor real-timely the frequency assignment and system running.

\subsection{Data Service Bus}

The data service bus is established on the base of communications network of wireless sensor network in dynamical frequency assignment system of WSN, which is in charge of transmission a large number of frequency information perceived by the node, reset information sent from cloud platform to the node, and, internal transactional communication in the cloud platform. A fixed point to point wired or wireless communication interface is held in the different regions of the data service bus respectively, with facilitating information exchange frequency between base stations and distribution systems. The results analyzed by cloud computing platform are transmitted from the service bus to the base station, what guides distributed detection sensor nodes to complete behavior of distributed detection and switching task of node frequency through the multiple independent, flexible control channel. Meanwhile, in order to satisfy the real-time requirements of frequency information transported, data service bus should support reliable ultra-low latency transmission in large scale nodes, and have highly efficient message ordering, synchronization, state migration, and fault tolerance, prevent failure of single points .

\section{3. cloud resource scheduling configuration}

Cloud computing virtualization technology is applied to integrate and call the abstracted base station hardware resource by the layer of resource scheduling configuration. Firstly, by use of cloud computing virtualization technology, number of physical resources in the bottom of distribution system (including hardware resources outside the base station) are abstracted virtual resources the same function and interface as physical resources. Then the resources are virtualized into $\mathrm{n}$ slots, and according to CPU type, operating system, memory size and so on, a resource pool is formed by the resources grouped. Finally, Multi-level resource pools are obtained. One of which is used to be manage and apply of virtual resource as cloud server, When there being a large number of spectrum sensing and frequency analysis tasks which will be divided into several parts, According to various parts of the virtual computing resources, it decides to perform each task of computing devices, and integrates the final results back to the node. According to virtualization of physical resources and dynamic assignment of computational load to complete the decomposition and distribution of sensory data, and achieve the integration of computing resources and distribution according to need.

\subsection{Real-time data memory}

In order to carry on the wireless sensor network's frequency data analysis and decision-making, in the WSN Dynamical frequency assignment system's real-time information database should be composed of many subdatabases, as shown in Table 1: 
Table 1. WSN dynamical frequency assignment system's database

\begin{tabular}{ll}
\hline \multicolumn{1}{c}{ Database name } & \multicolumn{1}{c}{ Data type and content } \\
\hline Frequency database & $\begin{array}{l}\text { Authorization frequency range, Protection frequency, Frequency assignment situation, } \\
\text { Frequency service condition and so on }\end{array}$ \\
$\begin{array}{l}\text { Transmition data rate of the node, acceptable error rate, Time delay limit, Transmission } \\
\text { mode, Bandwidth, Antenna model, Software radio module performance and so on }\end{array}$ \\
$\begin{array}{l}\text { Base station database } \\
\begin{array}{l}\text { Electromagnetic environment } \\
\text { database }\end{array}\end{array}$ & Base station's position, Server model, Network and working condition \\
\hline Geography database & Geography terrain feature, Latitude and longitude, Node distribution and so on \\
\hline Experience database & Includes the Former, Empirical data, Knowledge \\
\hline
\end{tabular}

In the WSN dynamic frequency assignment system's work process, massive real-time dynamic updating data is managed by the distributional storage technology of the cloud computating, i.e. the information resources are stored and dispatched through the computer clusters' way. By integrating the interdependent storage nodes such as base stations, the distributional storage system can effectively solve the problems deriving from the running process of dispatching system, e.g. gathered cognition information, massive real-time data and the storage and the process of cumulative massive date generated in the business procedures. The distributional file systems in the distributional storage system, e.g. GFS, HDFS, S3, can support the coexistence of Master and Slave in one or more databases, which can (1)dramatically improve the response speed and storage volume in the dispatching system, and (2)enhances the real-time features in frequency assignment by running the computation at the mission computation nodes, which can reduce the distance between computation running nodes and the data storage nodes and then reduce the data transmission time in the computing.

\subsection{Distributional policy-making support}

The complexity of frequency analysis, the real-time features of frequency assignment and massive frequency requirement of terminal nodes imposes very heavy duty to the WSN dynamic frequency assignment system. Distributional decision support system is the core of the whole distribution system. It provides the capability of processing, analyzing, computing massive real-time frequency data which can effectively support the running of the assignment system. It receives computation instructions from resource assignment layer, and by integrating computation resources, addresses the problems of massive computation in the assignment process of supporting system with both distributed computation and parallel computation approaches. The integrated computation engine makes gives powerful processing capability to assignment system, which can rapidly analyze and make decisions based on the spectrum cognition information received from terminals and transform it into reset information to massive frequency terminals.

The distributional decision-making support module is constituted by computation engines and strategy engines. The strategy engines consist of electromagnetic environment field,terminal node field,spectrum operation field,user field, etc. The frequency analysis mainly includes path LOS, error bit rate of radio link, time delay of link layer,interference,holding time of Channel, data analysis for channel capacity and so on. Based on the result of frequency analysis, the QoS demand of terminal nodes and decision-making principles, the frequency decision-making mode can carry out EMC analysis, boundary coordinated analysis,optimized computation, and finally transmit the reset information including Transmitter power, modulation patten, carrier frequency, dynamirange, antenna direction, expansion type and code. The whole process of spectrum analysis and frequency assignment is one process which could distinguish the electromagnetic environment change and self-adapt, as shown in Fig. 3. 


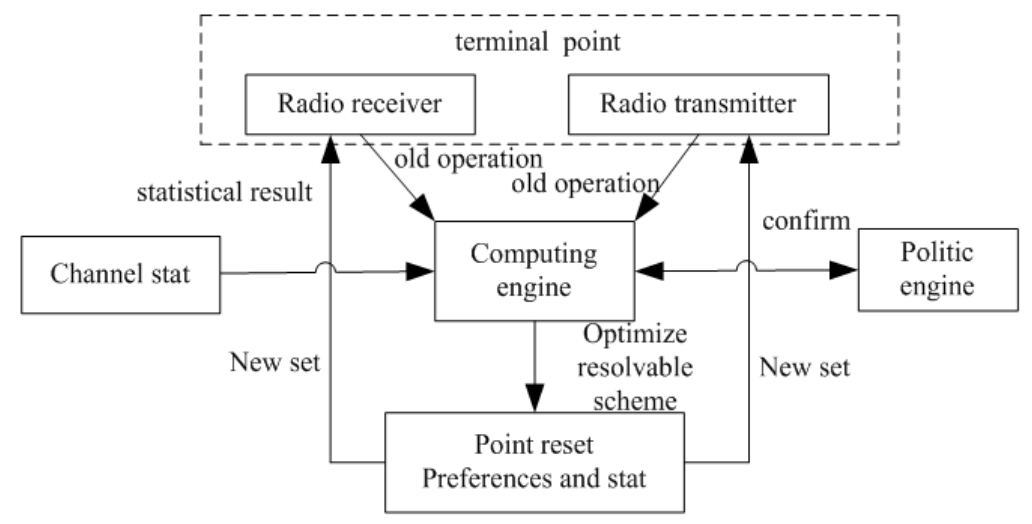

Fig. 3. The process of frequency adaptive control on DOSS

During the process of WSN dynamic frequency assignment, the assignment strategy in the frequency policy engine should make some real-time dynamic changes along with the variation of electromagnetic environment, communication mode, system parameter, network structure or node in the wireless sensor network. Spectrum assignment strategy is no longer a predetermined, fixed, but should have certain cognitive learning function, can change adaptively with the changes of time, space and frequency as well, thus can realize "strategy change agilely" finally. Using the SOAP protocol based on XML, as well as the knowledge of data service bus in cloud computing, distributed real-time data storage and adaptive decision support, etc., this paper established a prototype system of dynamic frequency assignment based on Web Services and WSN. The real-time dynamic frequency assignment hierarchy interface aiming at the wireless network structure is shown as the following Fig. 4.

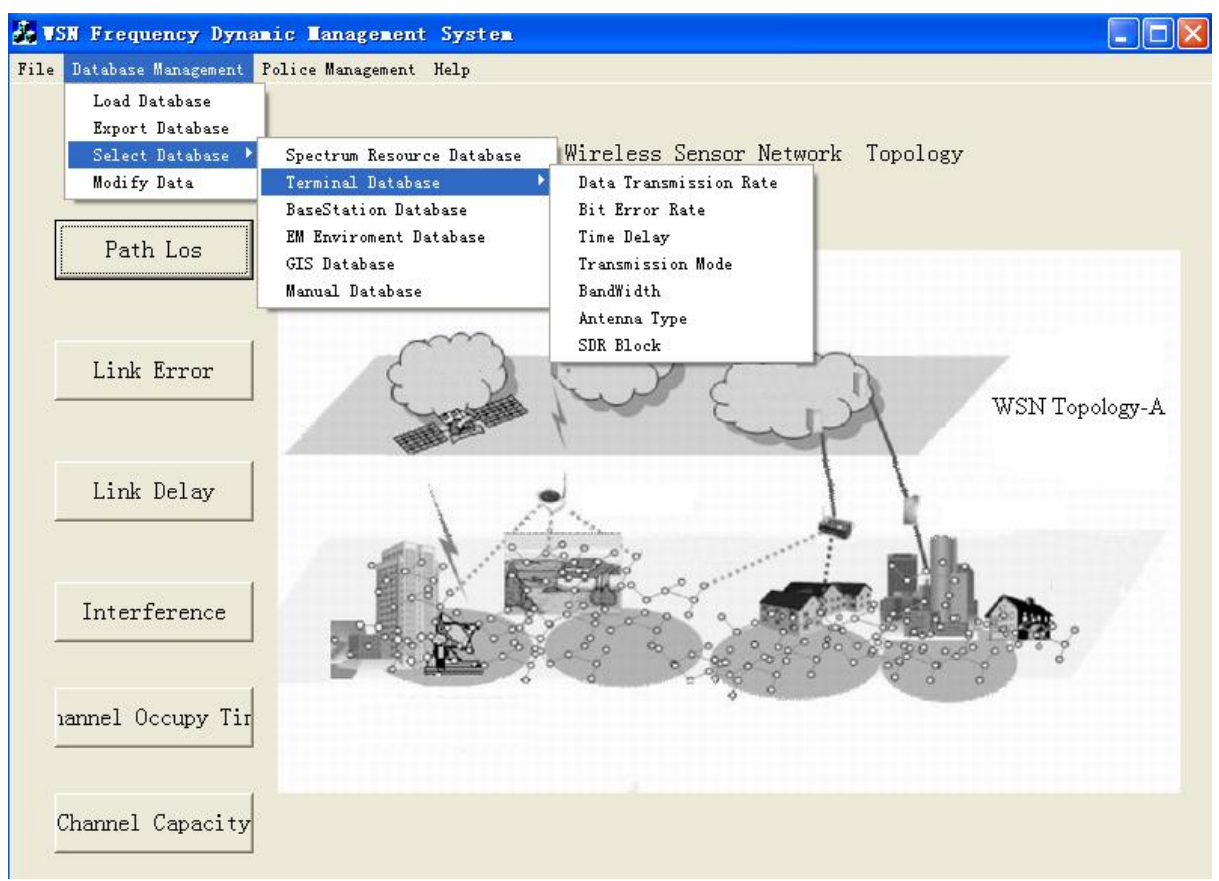

Fig. 3. WSN dynamic frequency allocation system 


\section{Conclusion}

Under the circumstance of significant contradiction between the lack of radio spectrum resource and the enormous demand of Wireless Sensors Networks. Study of Wireless Sensors Networks dynamic frequency assignment based on cloud computing is a new exploration to frequency assignment strategy. Using the data service bus, data memory, adaptive decision-making sertoli of cloud computing, achieving widely or large Wireless Sensors Networks distributed frequency assignment,giving a new thought for the efficient distribution of Wireless Sensors Networks.

\section{Acknowledgements}

This work is supported by National Natural Science Foundation Project (71071173) and Chongqing Soft Sciences Project(CSTC, 2010CE0109).

\section{References}

[1] Zhou Zhong, Zhou Shengli, Cui Shuguang, et al. Energy-efficient Cooperative Communication in a Clustered Wireless Sensor Network[J]. IEEE Trans. on Vehicular Technology, 2008, 57(6).

[2] FCC.ET Docket No 03-222 Notice of proposed rule making and order[R]. December, 2003.

[3] I F Akyildiz, W Y Lee, M C Vuran, "Next Generation/Dynamic Spectrum Access/Cognitive Radio Wireless Networks: A Survey,”Computer Networks Journal(Elsevier), 2009, 50(10):2127-2159.

[4] Junhua Zhu, Ka-Lok Hung, Brahim Bensaou , et al. "Rate-lifetime tradeoff for reliable communication in wireless sensor networks," Computer Networks vol. 52 ,2008, pp. 25-43.

[5] XG Working Group. The XG Vision Request For Comments Version 2.0[R], 2004.

[6] I. Chatzigiannakisa, E.Konstantinoub,V. Liagkoua, et al. "Design, Analysis and Performance Evaluation of Group Key Establishment in Wireless Sensor Networks,"Electronic Notes in Theoretical Computer Science vol 171, $2007,17-31$

[7] H.B. Chen, C. K. Tse, J.C. Feng, "Performance evaluation of source extraction in wireless sensor networks," Computer Communications vol 31, 2008, pp.3870-3875

[8] Daniel J A. Data management in the cloud: limitations and opportunities. Bulletin of the IEEE Computer Society Technical Committee on Data Engineering, 2009, 32(1):3 12. 